\title{
Tumor de los cordones sexuales con túbulos anulares ováricos asociado a síndrome de Peutz-Jeghers: reporte de un caso en la adolescencia
}

\author{
Tumor of the sexual cords with ovarian annular tubules associated with \\ Peutz-Jeghers syndrome in adolescence: a case report
}

\author{
Dra. Giselle T. Ponce , Dra. Cecilia Zunana ${ }^{a}$, Dra. Marianela Viso br Dra. María M. Bailezc, \\ Dra. Nilda González Roibon ${ }^{d}$ Dra. Andrea Bosaleh y Dra. Paula Califano ${ }^{a}$
}

\begin{abstract}
RESUMEN
Los tumores de los cordones sexuales y estromales son neoplasias poco frecuentes, que corresponden al $8 \%$ de los tumores primarios del ovario. El tumor delos cordones sexuales con túbulos anulares del ovario es considerado un subtipo y es infrecuente. Puede presentarse de manera esporádica o asociado al síndrome dePeutz-Jeghers y tiene diferente comportamiento y características en cada situación.

Se presenta el caso de una paciente adolescente con diagnóstico de tumor de los cordones sexuales con túbulos anulares del ovario asociado a síndrome de Peutz-Jeghers.

Palabras clave: tumores de los cordones sexuales y estroma de las gónadas, síndrome de Peutz-Jeghers, síndromes neoplásicos hereditarios.
\end{abstract}

\begin{abstract}
Tumors of the sexual and stromal cords are rare neoplasms, corresponding to $8 \%$ of primary ovarian tumors. The tumor of the sexual cords with annular tubules of the ovary is considered a subtype and is uncommon. It can occur sporadically or associated with Peutz-Jeghers Syndrome, having different behavior and characteristics in each situation.

We present the case of an adolescent patient with a diagnosis of a tumor of the sexual cords with annular tubules of the ovary associated with Peutz-Jeghers Syndrome.

Key words: sex cord-gonadal stromal tumors, Peutz-Jeghers syndrome, hereditary neoplastic syndromes.
\end{abstract}

http:/ / dx.doi.org/10.5546/ aap.2020.e329

Cómo citar: Ponce GT, Zunana C, Viso M, Bailez MM, et al. Tumor de los cordones sexuales con túbulos anulares ováricos asociado a síndrome de Peutz-Jeghers: reporte de un caso en la adolescencia. Arch Argent Pediatr. 2020;118(3):e329-e332.

a. Sección de Ginecología, Clínica Pediátrica.

b. Servicio de Hematooncología.

c. Servicios Quirúrgicos.

d. Servicio de Patología.

Hospital Nacional de Pediatría S. A. M. I. C. “Prof. Dr. Juan

P. Garrahan", Ciudad Autónoma de Buenos Aires.

Correspondencia:

Dra. Giselle T. Ponce: giseponce86@gmail.com

Financiamiento: Ninguno.

Conflicto de intereses: Ninguno que declarar.

Recibido: 21-8-2019

Aceptado: 7-11-2019

\section{INTRODUCCIÓN}

Los tumores de los cordones sexuales y estromales (TCSE) son neoplasias poco frecuentes, que corresponden al $8 \%$ de los tumores primarios del ovario. ${ }^{1}$ El tumor de los cordones sexuales con túbulos anulares del ovario (TCSTA) es considerado un subtipo y constituye una entidad infrecuente compuesta por células del cordón sexual que conforman túbulos anulares. Puede presentarse de manera esporádica o asociado al síndrome de Peutz-Jeghers (SPJ) ${ }^{2}$ y tiene diferente comportamiento y características en cada caso. El manejo es quirúrgico, con preservación o no de la fertilidad, según el estadio, la edad y el deseo de la paciente.

\section{CASO CLÍNICO}

Paciente femenina de 13 años y 7 meses de edad con antecedente de invaginación intestinal y resección de pólipos hamartomatosos de tipo Peutz-Jeghers de localización gástrica y colónica, asociados a una pigmentación mucocutánea en el labio, por lo que se realizó el diagnóstico clínico de SPJ. Era la quinta hija de una pareja no consanguínea, con cuatro hermanos mayores sin historia familiar compatible con SPJ, por lo que pudo corresponder a un caso esporádico.

Adolescente con menarca a los 12 años, ciclos regulares y examen genital normal. Identificación acorde al género, orientación heterosexual sin inicio de relaciones sexuales. Se solicitó una ecografía ginecológica por dismenorrea leve y se evidenció una imagen anexial izquierda (Figura 1). Se solicitaron marcadores tumorales séricos, que mostraron antígeno de cáncer 125 (cancer antigen 125; $C A-125$, por sus siglas en inglés): $24,5 \mathrm{U} / \mathrm{ml}$; antígeno carcinoembrionario (carcinoembryonic antigen; CEA, por sus siglas en inglés): $0,7 \mathrm{ng} / \mathrm{ml}$; alfa feto-proteína: $1,8 \mathrm{U} / \mathrm{ml}$; subunidad beta de la gonadotropina coriónica humana (beta subunit of human chorionic gonadotropin; $h C G \beta$, por sus siglas en inglés) 
FiguRA 1. Ecografía ginecológica. Formación anexial izquierda hiperecogénica, redondeada, de bordes bien definidos, con centro más hipoecoico, ligero refuerzo acústico posterior y vascularización en su interior de 2,4 $x 2,4 \times 1,8 \mathrm{~cm}$, sugestiva de quiste dermoide

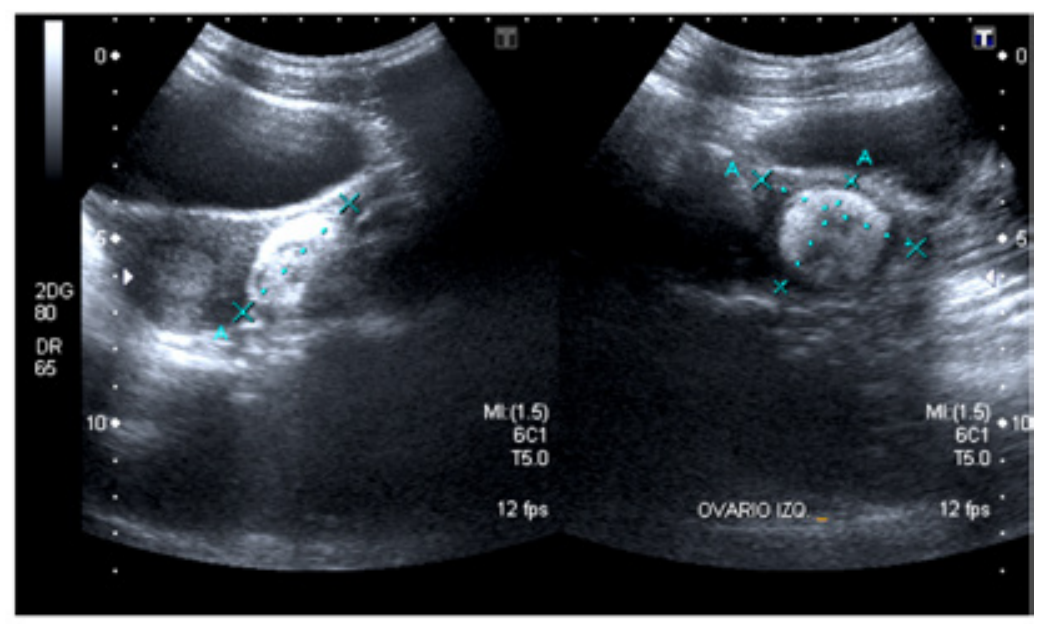

Figura 2. Macroscópica intraoperatoria. Durante el acto quirúrgico, se observa macroscópicamente una formación nodular de $3 \times 3 \times 2 \mathrm{~cm}$, con superficie externa irregular. Al corte, tejido sólido, amarillento

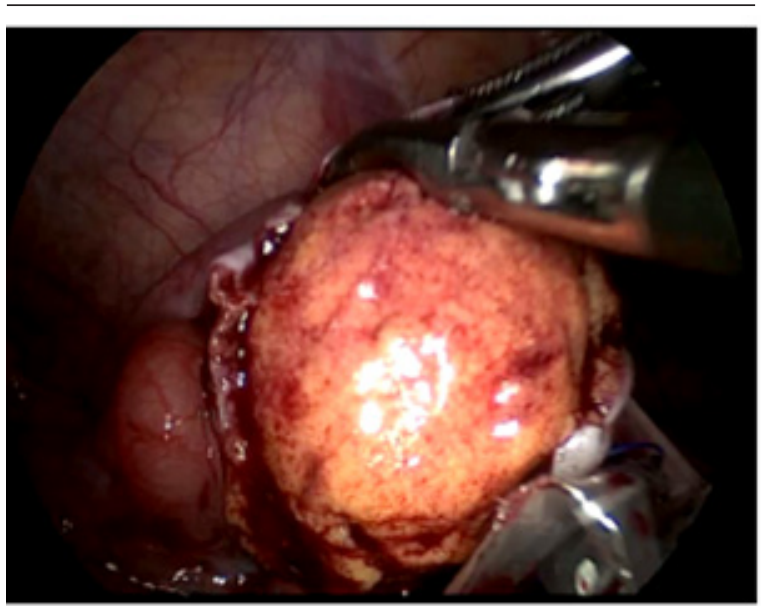

cuantitativa negativa; lactato deshidrogenasa (LDH): $366 \mathrm{UI} / 1$; todos dentro de los valores normales (VN), a excepción de la inhibina B de $350 \mathrm{pg} / \mathrm{ml}$ (VN < $175 \mathrm{pg} / \mathrm{ml}$ para la edad).

Se realizó la exéresis laparoscópica de la formación con conservación del ovario (Figura 2). En el examen microscópico, se observó una proliferación organizada en túbulos complejos rodeados de membrana basal gruesa, eosinofílica que contenía luces con cuerpos hialinos. Estroma fusocelular blando. Presencia de hasta 1 mitosis / campos de gran aumento (CGA) y múltiples calcificaciones. Sin necrosis (Figura 3). Las células mostraron expresión difusa con patrón de membrana de CD99, inhibina y creatina quinasa (creatine kinase; $C K$, por sus siglas en inglés) (AE1-AE-3). No se observó marcación con antígeno epitelial de membrana (epithelial membrane antigen; EMA, por sus siglas en inglés).

Figura 3. A. Microscopía. H-E40X. Proliferación organizada en túbulos anulares complejos. B. Microscopía. H-E100X. Células de Sertoli dispuestas en túbulos complejos constituidos por células pálidas en torno a cuerpos hialinos y áreas calcificadas
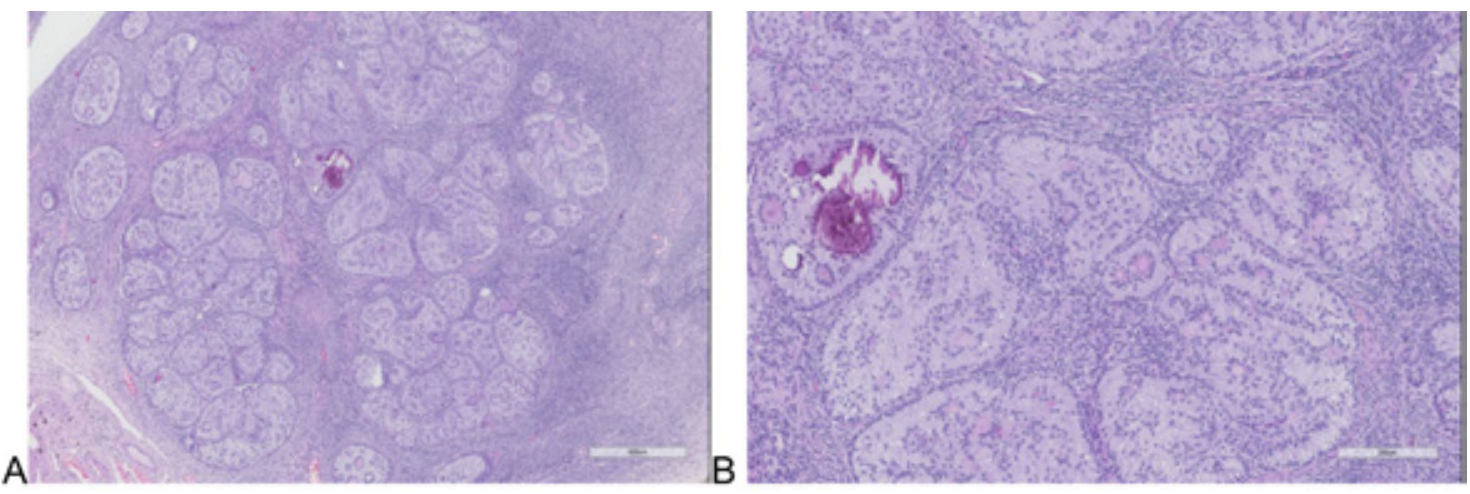
Diagnóstico: tumor de los cordones sexuales con túbulos anulares del ovario. Márgenes quirúrgicos en contacto con la lesión. Se realizó una nueva exploración quirúrgica laparoscópica: cavidad abdominal, útero y anexo derecho, macroscópicamente no comprometidos. Se llevó a cabo una anexectomía izquierda, y se identificó una lesión residual microscópica de $0,5 \times 0,3 \mathrm{~cm}$, con iguales características histológicas e inmunohistoquímicas que la descritas en la biopsia original. Se realizaron estudios de estadificación, sin evidencia de enfermedad (tumor localizado). Se solicitaron estudios complementarios a sus padres y un estudio genético a la paciente, los cuales están pendientes. Se brindó asesoramiento sobre el diagnóstico, pronóstico, patrón de herencia e importancia del seguimiento interdisciplinario a la paciente y su familia.

\section{DISCUSIÓN}

El SPJ es una enfermedad autosómica dominante caracterizada por la presencia de múltiples pólipos hamartomatosos en el tracto gastrointestinal, pigmentación mucocutánea e incremento de riesgo de cáncer hereditario (Tabla 1). Es una entidad infrecuente con una prevalencia estimada de $1: 8000$ a $1: 200000$, con igual afectación en hombres y mujeres. Está asociado con la mutación en el gen STK11, con función supresora de tumores, ubicado en el cromosoma 19p13.3, que codifica una serinatreonina quinasa.

Aproximadamente, un $25 \%$ tienen una mutación de novo. ${ }^{3}$ El riesgo de cáncer gastrointestinal oscila entre el $37 \%$ y el $93 \%$. Se asocia con tumores extracolónicos, mayormente, en el páncreas y la mama. Las mujeres tienen un riesgo incrementado de adenocarcinoma de desviación mínima (neoplasia de cérvix no relacionada con el virus del papiloma humano -human papillomavirus; HPV, por sus siglas en inglés-) con características histológicas de aspecto benigno, que constituyen un desafío diagnóstico, ${ }^{4}$ y neoplasias ováricas. ${ }^{5}$ Existen diferentes recomendaciones para el seguimiento ginecológico, que incluyen examen mamario desde los 18 años, mamografía (se debe considerar la resonancia magnética nuclear-RMN-) desde los 25 años, ecografía ginecológica y $C A-125$ desde los 18 o 25 años (según las series) y realización de Papanicolaou. ${ }^{6-9}$

Aproximadamente, $1 / 3$ de los TCSTA está asociado a SPJ. ${ }^{10}$ La primera descripción de esta entidad fue hecha por Scully en $1970 .{ }^{11}$ Suelen tener comportamiento benigno, aunque los casos no asociados a SPJ tienen mayor tendencia a la malignidad. ${ }^{12}$ Ocurren, mayormente, en la edad reproductiva (promedio de 30 años, ${ }^{13}$ aunque se han descrito casos en pacientes posmenopáusicas (rango: 4-76 años). ${ }^{14}$ Pueden ser productores de estrógeno (el 40-60\%) y manifestarse con pseudopubertad precoz, sangrado genital o, si hay componente androgénico, producir hirsutismo, hipertrofia de clítoris y cambios en la voz. En los casos en los que no hay producción hormonal, la clínica es de dolor abdominal. ${ }^{13}$ Las formas esporádicas suelen presentarse en la cuarta década, asociadas a sintomatología por secreción de estrógenos, ser unilaterales y grandes. Por el contrario, los TCSTA asociados a SPJ suelen presentarse incidentalmente en la tercera década y ser bilaterales y pequeños. ${ }^{15}$

En nuestro caso, se trató de un hallazgo

TABLA 1. Riesgo individual de neoplasias extracolónicas asociadas a síndrome de Peutz-Jeghers, comparado con el riesgo de población general estimado y el rango de edad promedio al momento del diagnóstico

\begin{tabular}{lccc}
\hline Localización & $\begin{array}{c}\text { Riesgo de } \\
\text { la población general }\end{array}$ & $\begin{array}{c}\text { Riesgo } \\
\text { sindrómico }\end{array}$ & $\begin{array}{c}\text { Rango de edad promedio al } \\
\text { momento del diagnóstico }\end{array}$ \\
\hline Estómago & $<1 \%$ & $29 \%$ & $30-40$ \\
Intestino delgado & $<1 \%$ & $13 \%$ & $37-42$ \\
Páncreas & $1,5 \%$ & $11-36 \%$ & $41-52$ \\
Mama & $12,4 \%$ & $32-54 \%$ & $37-59$ \\
Ovario (mayormente TCSTA) & $1,6 \%$ & $21 \%$ & 28 \\
Útero & $2,7 \%$ & $9 \%$ & 43 \\
Cervix (adenoma maligno) & $<1 \%$ & $10 \%$ & $34-40$ \\
Testículo (tumor de Sertoli) & $<1 \%$ & $9 \%$ & $6-9$ \\
Pulmón & $6,9 \%$ & $7-17 \%$ & 47 \\
\hline
\end{tabular}

TCSTA: tumor de los cordones sexuales con túbulos anulares.

Adaptado de Syngal S, Brand RE, Church JM, et al. Am J Gastroenterol. 2015;110(2):223-263. ${ }^{3}$ 
incidental de un tumor pequeño, pero unilateral. Con independencia del contexto clínico, histológicamente, muestran las mismas características y presentan estructuras tubulares simples y complejas, constituidas por células de citoplasma amplio pálido ${ }^{13}$ con la marcación descrita en nuestro caso. Los tumores esporádicos suelen formar una masa tumoral, mientras que, en los TCSTA asociados a SPJ, los túbulos anulares se distribuyen en el estroma ovárico, sin constituir una formación tumoral. Usualmente, se identifican 3-4 figuras mitóticas en 10 CGA. No existen criterios histológicos definitivos de malignidad, y es difícil predecir su comportamiento. Un patrón de crecimiento infiltrativo e incremento de la actividad mitótica han sido asociados a un comportamiento más agresivo. $^{13}$

Respecto del tratamiento, los TCSTA con o sin SPJ son manejados con cirugía: anexectomía uni-, bilateral o asociada a histerectomía según el estadio, edad y deseos de fertilidad. Si el tumor tiene un comportamiento clínico maligno, se debe evaluar la necesidad de quimioterapia y/o radioterapia. Asimismo, como la vía de diseminación es fundamentalmente linfática, se puede plantear la realización de linfadenectomía pélvica para prevenir recurrencias. Sin embargo, no hay protocolos de tratamiento adyuvante, debido a la falta de datos en la literatura respecto del comportamiento y pronóstico de estos tumores. ${ }^{12}$

A pesar de su habitual comportamiento y aspecto histológico benigno, deben considerarse como una neoplasia potencialmente maligna, en especial, en los casos esporádicos, y, por tanto, efectuar un estrecho seguimiento imagenológico y con marcadores tumorales. Siendo imprescindible el trabajo interdisciplinario, se recomienda el seguimiento ginecológico desde la pubertad para realizar un control oportuno que permita diagnosticar tempranamente patologías asociadas, lo que mejora el pronóstico y la sobrevida de las pacientes.

\section{REFERENCIAS}

1. Lim D, Oliva E. Ovarian sex cord-stromal tumours: an update in recent molecular advances. Pathology. 2018; 50(2):178-89.

2. Young R. Ovarian Sex Cord-Stromal Tumors: Reflections on a 40-Year Experience With a Fascinating Group of Tumors, Including Comments on the Seminal Observations of Robert E. Scully, MD. Arch Pathol Lab Med. 2018; 142(12):1459-84.

3. Syngal S, Brand RE, Church JM, Giardiello FM, et al. ACG clinical guideline: Genetic testing and management of hereditary gastrointestinal cancer syndromes. Am J Gastroenterol. 2015; 110(2):223-63.

4. Banno K, Kisu I, Yanokura M, Masuda K, et al. Hereditary gynecological tumors associated with Peutz-Jeghers syndrome (Review). Oncol Lett. 2013; 6(5):1184-8.

5. Achatz MI, Porter C, Brugières L, Druker H, et al. Cancer Screening Recommendations and Clinical Management of Inherited Gastrointestinal Cancer Syndromes in Childhood. Clin Cancer Res. 2017; 23(13):e107-14.

6. Khanna K, Khanna V, Bhatnagar V. Peutz-Jeghers syndrome: need for early screening. BMJ Case Rep. 2018; 11(1):e225076.

7. Riegert-Johnson DL, Gleeson FC, Westra W, Hefferon T, et al. Peutz-Jeghers Syndrome. In: Riegert-Johnson DL, Boardman LA, Hefferon T, Roberts M (eds.). Cancer Syndromes. Bethesda (MD): National Center for Biotechnology Information; 2009.Pág.26.

8. Beggs AD, Latchford AR, Vasen HF, Moslein G, et al. Peutz Jeghers syndrome: asystematicreview and recommendations for management. Gut. 2010; 59(7):975-86.

9. Turpin A, Cattan S, Leclerc J, Wacrenier A, et al. Prédisposition héréditaire aux cancers digestifs, mammaires, gynécologiques et gonadiques: état des lieux du syndrome de Peutz-Jeghers. Bull Cancer. 2014; 101(9):813-22.

10. Oliva E, Álvarez T, Young RH. Sertoli Cell Tumors of the Ovary: a clinicopathologic and immunohistochemical study of 54 cases. Am J Surg Pathol. 2005; 29(2):143-56.

11. Scully RE. Sex cord tumor with annular tubules a distinctive ovarian tumor of the Peutz-Jeghers syndrome. Cancer.1970; 25(5):1107-21.

12. Chatziioannidou K, Botsikas D, Tille JC, Dubuisson J. Preservation of fertility in non-Peutz-Jegher syndromeassociated ovarian sex cord tumour with annular tubules. BMJ Case Rep. 2015; 2015:bcr2014207841.

13. Kurman RJ, Carcangiu ML, Herrington CS, Young RH. Sex cord-stromal tumours. In: WHOClassification of Tumours of the Female Reproductive Organs. 4th ed. Lyon: IARC Press; 2014.Págs.44-56.

14. Gupta N, Kothari DC, Arora N. Ovarian Sertoli Cell Tumor: A Rare Case of Sex Cord Stromal Tumor. Int J Sci Stud. 2014; 2(8):280-3.

15. Meserve E, Nucci M. Peutz-Jeghers Syndrome: Pathobiology, Pathologic Manifestations, and Suggestions for Recommending Genetic Testing in Pathology Reports. Surg Pathol Clin. 2016; 9(2):243-68 